\title{
Sistematização da assistência de enfermagem no pós-operatório de cirurgia cardíaca
}

\author{
Patrícia Flores de Oliveira de Aguiar*, Alex de Souza Pereira*, Messias Ferreira Barbosa*, Sabrine Rosa Dutra*, \\ Fernanda Garcia Bezerra Góes, M.Sc.**
}

"Acadêmicos de Enfermagem do Centro Universitário Plínio Leite (UNIPLI), **Enfermeira, Professora e Orientadora do Centro Universitário Plínio Leite (UNIPLI)

\section{Resumo}

O objetivo deste estudo foi conhecer a importância e os benefícios para os clientes com a implantaçáo da sistematização da assistência de enfermagem (SAE) no pós-operatório de cirurgia cardíaca. A metodologia utilizada é exploratória e qualitativa, realizada através da revisão sistemática de literatura com levantamento de dados na BVS (Lilacs e BDENF). Após coleta dos dados, foi realizada uma leitura seletiva, em que selecionamos 10 bibliografias potenciais. Ao realizar a leitura interpretativa e análise temática do material coletado, concluímos que há duas categorias: os benefícios e as dificuldades encontradas com o uso do SAE. Com isso percebemos a importância da sistematização da assistência de enfermagem para o cliente, como meio de criar possibilidades para obtermos uma assistência voltada às suas necessidades, proporcionando uma recuperação rápida e plena. Entretanto, é necessário que exista preparo destes profissionais nas universidades, e que as instituiçôes de saúde deem condiçóes para o seu desenvolvimento diminuindo desta forma a sobrecarga destes profissionais.

Palavras-chave: revascularização miocárdica, cirurgia cardíaca, processos de enfermagem, assistência de enfermagem, cuidados pós-operatórios.

\section{Abstract \\ Nursing care systematization in postoperative cardiac surgery}

The objective of this study was to know the importance and benefits of the systematization of nursing care (SNC) after cardiac surgery for the client. The methodology used an exploratory and qualitative approach, and a systematic literature review on BVS (Lilacs and BDENF) data base was realized. After data collection was carried out, an exploratory and selective reading was performed, and we selected 10 potential bibliographies. After an interpretive reading of this material and conducting a thematic analysis of the collected material, we concluded there are two categories: the benefits and the difficulties of using the SNC. Thus we have noticed how important it is the systematization of nursing care for the client, creating opportunities and assistance adapted to their needs, providing a quick and full recovery. The professional training in the universities and health institutions should give conditions for this development, consequently reducing workload of the professionals.

Key-words: myocardial revascularization, cardiac surgery, nursing process, nursing care, postoperative care. 


\section{Resumen}

\section{Sistematización de los cuidados de enfermería en postoperatorio de cirugía cardíaca}

El objetivo de este estudio es conocer la importancia y los beneficios para los clientes con la implementación de la sistematización de los cuidados de enfermería en postoperatorio de cirugía cardíaca. La investigación podrá contribuir para resaltar la importancia de esta sistematización de atención dispensada al cliente después de cirugía cardiaca, con el objetivo de describir los beneficios a los clientes sometidos a la sistematización de cuidados de enfermería en el postoperatorio de cirugía cardíaca. La metodología es de naturaleza exploratoria y cualitativa, realizada a través de una revisión sistemática de la literatura en la base de datos BVS (Lilacs y BDENF). Después de recolectar los datos, se realizó una lectura selectiva, y se seleccionaron 10 artículos importantes. Al terminar la lectura interpretativa y el análisis temático del material recolectado, se concluyó que hay dos categorías: beneficios y dificultades con el uso de la SCE. Con esto nos damos cuenta de la importancia de la sistematización de los cuidados de enfermería para el cliente, como un medio de crear oportunidades para obtener asistencia dirigida a sus necesidades, proporcionando una recuperación rápida y completa. Sin embargo, es necesario que las universidades preparen estos profesionales y que las instituciones de salud les ofrezcan condiciones para su desarrollo y, de esta manera, reducir la sobrecarga de trabajo de estos profesionales.

Palabras-clave: revascularización miocárdica, cirugía cardiaca, procesos de enfermería, atención de enfermería, cuidados postoperatorios.

\section{Introdução}

Com Florence Nightingale, em 1859, a enfermagem iniciou sua caminhada para a adoção de uma prática baseada em conhecimentos científicos, abandonando gradativamente a postura de atividade caritativa, intuitiva e empírica. Com esse intuito, diversos conceitos, teorias e modelos específicos da enfermagem foram e estão sendo desenvolvidos, com a finalidade de prestar assistência, ou seja, planejar as açóes, determinar e gerenciar o cuidado, registrar tudo o que foi planejado e executado e, finalmente, avaliar estas condições. Permitindo, assim, gerar conhecimentos a partir da prática e do processo da enfermagem, tornando o cuidado mais amplo [1]. Na década de 70, Wanda de Aguiar Horta desenvolveu um modelo conceitual, no qual a própria vivência na enfermagem levou-a a desenvolver um modelo que pudesse explicar a natureza da enfermagem, definir seu campo de ação específico e sua metodologia. Essa mesma autora define o processo de enfermagem como sendo a dinâmica das açóes sistematizadas e inter-relacionadas, visando à assistência ao ser humano [2].

A lei 7.498, de 25 de junho de 1986, referente ao exercício da enfermagem, dispóe em seu artigo 11 , como atividades exclusivas do enfermeiro a consulta de enfermagem; prescrição da assistência de enfermagem; os cuidados diretos de enfermagem a pacientes graves com risco de vida; até mesmo cuidados de enfermagem de maior complexidade e que exijam conhecimentos de base científica e capacidade de tomar decisóes imediatas referentes aos pacientes [3]. Como nos descreve a lei mencionada, compete ao enfermeiro a criaçáo de um plano de cuidados específicos, de acordo com as necessidades de cada cliente.

A sistematização da assistência de enfermagem (SAE) é uma metodologia para organizar e sistematizar o cuidado, com princípios do método científico que identificam as situaçóes de saúde, doenças e as necessidades de cuidados em enfermagem, visando não apenas a sua patologia de base, mas também a criar formas de intervir junto ao cliente para prevenir, promover, recuperar e reabilitar a sua saúde, bem como da família e da comunidade [4].

Esta é uma metodologia privativa do enfermeiro, que desenvolve açóes, adequando-as de acordo com as necessidades e individualidades dos clientes. A SAE, como também é conhecida, proporciona cuidados individualizados e cria oportunidades de gerar aos indivíduos qualidade no seu atendimento [4]. Com sua implementação ela torna possível a operacionalizaçáo dos processos de enfermagem que trata de um instrumento metodológico, servido como orientaçáo para o cuidado do profissional de enfermagem e para documentação de sua prática profissional. 
A adoção de sistemas de classificação permite o uso de uma linguagem única e padronizada, sendo igualmente interpretada em qualquer lugar, favorecendo o processo de comunicaçáo e a compilação de dados para o planejamento da assistência e seu desenvolvimento [5] Para isso, podemos fazer uso do livro de diagnósticos de enfermagem da North American Nursing Association (NANDA), que se define como um julgamento clínico sobre as respostas dos indivíduos, família, comunidade, aos problemas de saúde, processos vitais reais ou potenciais.

O diagnóstico de enfermagem visa lograr o alcance de resultados pelo qual o enfermeiro é responsável, pela prescrição da assistência de enfermagem, elaboração de um plano de cuidados onde são levantados pontos que atendam às necessidades do paciente, evolução da assistência de enfermagem que são as anotaçôes dadas como necessárias ao decorrer do tratamento e importantes para a elaboração dos cuidados de enfermagem [6].

A possibilidade de tornar o cuidado de enfermagem mais individualizado, que atenda às reais necessidades dos indivíduos, tornando-se o diferencial da assistência foi um dos pontos que nos motivaram a realizar esta pesquisa, bem como os altos índices de pessoas com problemas coronarianos.

Esse aumento na incidência de cirurgias cardíacas nos últimos anos ocorre não apenas por fatores hereditários, mas também pelo estilo de vida adotado nos dias atuais, podendo ocorrer com qualquer indivíduo, dos mais variados níveis socioeconômicos e etários, e podem se agravar no pós-operatório devido à falta de um plano de cuidados adequado para a sua história patológica e de vida.

Pensando nisso, e levando em consideração o que foi vivenciado por nós, enquanto acadêmicos de enfermagem, durante os estágios, percebemos que, em algumas situaçóes, não se dava a atenção necessária ao atendimento das necessidades individuais dos clientes. Porém, acreditávamos que com o uso da SAE seria possível elaborar um plano de cuidados diferenciados para cada cliente, em que ele tivesse suas necessidades atendidas, com os riscos de agravos diminuídos, tornando, assim, a assistência individualizada e eficiente.

Esta pesquisa procura evidenciar que, com o uso da SAE, é possível não só identificar e dar atenção a diversos fatores como doenças crônicas pré-existentes (ansiedades, problemas de níveis sociais afastamento da família e dos amigos por um período), como fazer o controle destes fatores. Essa metodologia facilita o pós-operatório e contribui para que a enfermagem se afaste do paradigma de que apenas recupera e reabilita, assumindo um caráter de prevenção e promoção à saúde.

No Brasil, aproximadamente vinte e quatro mil e quinhentas cirurgias cardíacas são realizadas anualmente e, nos últimos anos, numerosas mudanças aconteceram nas condutas dos pós-operatórios imediatos que influenciam a velocidade de recuperação da cirurgia cardíaca. Os avanços tecnológicos na Circulação Extracorpórea (CEC), melhora na proteçấo miocárdica. $\mathrm{O}$ aparecimento de procedimentos minimamente invasivos e a disponibilidade de agentes anestésicos de curta duração permitem uma recuperaçáo ainda mais rápida [7].

Como toda cirurgia de grande porte, ela é realizada com o uso da anestesia geral, e por este motivo é importante o conhecimento das alterações hemodinâmicas determinadas pelas doenças cardíacas, assim como a interação com agentes anestésicos. Os fatores que desequilibram a homeostase cardíaca, como a hipotensão, anemia, hipovolemia, uso indiscriminado de agentes inotrópicos e vasopressores, devem ser evitados ou corrigidos [7].

O bom resultado da cirurgia cardíaca se baseia não somente nos detalhes técnicos intra-operatórios, mas também em condutas adequadas no período pós-operatório, período este que, na maioria dos pacientes, caracteriza-se por um padrão típico de alteraçóes fisiopatológicas e, portanto, o seu conhecimento prévio nos possibilita uma melhor padronização de medidas a serem tomadas [8].

Devido a todos estes pontos discutidos sobre a SAE, e a necessidade de se ter um acompanhamento mais de perto deste cliente em pós-operatório de cirurgia cardíaca, identificamos que o nosso objetivo era conhecer a importância e os benefícios para os clientes com a implantação da sistematização da assistência de enfermagem no pós-operatório de cirurgia cardíaca.

Sabe-se que o atendimento diferenciado é aquele que consegue atender as necessidades humanas básicas, sem deixar de prestar a atenção necessária na sua atual condição, acompanhando todo o processo de reabilitação e intervindo quando necessário. Esse diferencial torna-se importante para que haja a integralidade do cuidado, pois estabelecemos uma linha comum que auxilia os enfermeiros na avaliação dos dados selecionados, na identificação e na descrição dos problemas potenciais ou reais do cliente [4]. 
Alguns estudos mostram que houve uma mudança progressiva no tipo de paciente que é submetido à cirurgia cardíaca. Cada vez mais a idade média dos pacientes, a gravidade das lesóes coronarianas, o grau de disfunção e as patologias associadas, bem como a incidência de cirurgias de emergência, têm aumentado, levando a uma maior dificuldade no manuseio dos pacientes devido à complexidade desses novos casos [7].

O período pós-operatório imediato para o paciente que sofreu cirurgia cardíaca apresenta muitos desafios para a equipe de saúde. Todos os esforços são feitos para facilitar a transição da sala de cirurgia para a unidade de cuidados pós-anestésicos com riscos mínimos. As informaçôes específicas sobre a cirurgia e os importantes fatores sobre o tratamento pós-operatório são comunicados pela equipe cirúrgica e da anestesia ao enfermeiro de cuidados críticos que, em seguida, assume a responsabilidade pelos cuidados ao paciente [1]. Estas informaçóes constituem dados importantes, desde o momento da chegada do paciente a UTI. Assim, detalhes sobre o transoperatório e eventuais intercorrências, os balanços hídricos e sanguíneo, o tempo de perfusão e de clampeamento aórtico durante o ato cirúrgico são dados fundamentais [7].

Apesar de a SAE estar incorporada à prática de enfermagem e estar em alta, algumas instituiçóes ainda náo utilizam este recurso como instrumento de cuidado.

O objetivo que norteia esta pesquisa é descrever os benefícios da implantação da sistematização da assistência de enfermagem no pós-operatório de cirurgia cardíaca para os clientes.

Ressalta-se que esta pesquisa pretende contribuir para destacar a importância da sistematizaçáo da assistência de enfermagem, no que diz respeito aos cuidados dispensados no cliente submetido à cirurgia cardíaca, de forma integral. Destacando, com isso, vários aspectos importantes à sua rápida recuperação e para que este período náo seja acometido por complicaçóes por falta de um acompanhamento mais detalhado de suas necessidades. Além de contribuir para futuras pesquisas e com o ensino na área.

\section{Material e métodos}

O presente estudo foi realizado das seguintes formas: de natureza exploratória, quando não existem elementos ou dados suficientes para o pesquisador [9]; através da revisão sistemática de literatura, que tem por objetivo principal trazer veracidade aos fatos lançados no trabalho [9]; através de um levantamento de dados na internet, utilizando como descritores: revascularização miocárdica, cirurgia cardíaca, processos de enfermagem, assistência de enfermagem, cuidados pós-operatórios; de toda a produção científica em português encontrada na Biblioteca Virtual de Saúde (BVS); nas bases de dados: Lilacs e BDENF.

A abordagem utilizada foi qualitativa, que são informaçóes obtidas que não podem ser quantificáveis e os dados são analisados intuitivamente [10].

Este estudo foi sistematizado e desenvolvido com base em material publicado por meio de consultas em artigos científicos, livros especializados e revistas, onde se buscou um aprofundamento sobre a temática de maneira pela qual fosse possível entender as diferentes formas de contribuição científica sobre o assunto.

Inicialmente realizamos uma pesquisa utilizando os descritores isoladamente, como no Quadro 1:

Quadro 1 - Dados quantitativos referentes aos descritores isolados.

\begin{tabular}{|l|c|c|c|}
\hline \multicolumn{1}{|c|}{ Descritor } & Lilacs & BDENF & Total \\
\hline Assistência de Enfermagem & 2635 & 2046 & 4681 \\
\hline Cirurgia Cardíaca & 1048 & 60 & 1108 \\
\hline Processos de Enfermagem & 373 & 294 & 667 \\
\hline Revascularização Miocárdica & 903 & 10 & 913 \\
\hline Cuidados pós-operatório & 1097 & 132 & 1229 \\
\hline
\end{tabular}

Devido ao grande quantitativo de material encontrado, resolveu-se associar os descritores em dupla e depois todos os descritores juntos, com a finalidade de refinar a pesquisa, como pode ser observado no Quadro 2.

Quadro 2 - Associação dos descritores.

\begin{tabular}{|l|c|c|c|}
\hline \multicolumn{1}{|c|}{ Descritor } & Lilacs & BDENF & Total \\
\hline $\begin{array}{l}\text { Assistência de Enfermagem + } \\
\text { Cirurgia Cardíaca }\end{array}$ & 19 & 12 & 31 \\
\hline $\begin{array}{l}\text { Assistência de Enfermagem + } \\
\text { Processos de Enfermagem }\end{array}$ & 93 & 82 & 205 \\
\hline $\begin{array}{l}\text { Assistência de Enfermagem + } \\
\text { Revascularização Miocárdica }\end{array}$ & 0 & 0 & 0 \\
\hline $\begin{array}{l}\text { Assistência de Enfermagem + } \\
\text { Cuidados Pós Operatórios }\end{array}$ & 0 & 0 & 0 \\
\hline $\begin{array}{l}\text { Cirurgia Cardíaca + Proces- } \\
\text { sos de Enfermagem }\end{array}$ & 0 & 0 & 0 \\
\hline $\begin{array}{l}\text { Cirurgia Cardíaca + Revas- } \\
\text { cularização Miocárdica }\end{array}$ & 53 & 3 & 56 \\
\hline
\end{tabular}




\begin{tabular}{|l|c|c|c|}
\hline $\begin{array}{l}\text { Cirurgia Cardíaca + Cuida- } \\
\text { dos Pós Operatórios }\end{array}$ & 0 & 0 & 0 \\
\hline $\begin{array}{l}\text { Processos de Enfermagem + } \\
\text { Revascularização Miocárdica }\end{array}$ & 1 & 0 & 1 \\
\hline $\begin{array}{l}\text { Processos de Enfermagem + } \\
\text { Cuidados Pós Operatórios }\end{array}$ & 0 & 0 & 0 \\
\hline $\begin{array}{l}\text { Revascularização Miocárdica } \\
+ \text { Cuidados Pós Operatórios }\end{array}$ & 0 & 0 & 0 \\
\hline $\begin{array}{l}\text { Assistência de Enfermagem } \\
+ \text { Cirurgia Cardíaca + } \\
\text { Processos de Enfermagem + } \\
\text { Revascularização Miocárdica } \\
+ \text { Cuidados Pós Operatório }\end{array}$ & 0 & 0 & 0 \\
\hline
\end{tabular}

A partir deste refino, foram selecionados 10 artigos, para a construção desta pesquisa, conforme apresenta o Quadro 3.

Foram usados como critério de exclusão: Artigos escritos há mais de dez anos; e artigos que menos se relacionavam com o tema da pesquisa.

Quadro 3 - Bibliografia potencial.

\begin{tabular}{|c|c|c|c|}
\hline Autores & Ano & Tífulo & Base de dados \\
\hline $\begin{array}{l}\text { Guerriero ALS, Guimarães } \\
\text { HCQCP, Maria VLR [ } 111] .\end{array}$ & 2000 & $\begin{array}{l}\text { Diagnóstico de enfermagem do paciente adulto } \\
\text { no primeiro pós-operatório de cirurgia cardíaca }\end{array}$ & $\begin{array}{l}\text { Lilacs; Acta Paul Enferma- } \\
\text { gem 13(2):59-67. }\end{array}$ \\
\hline $\begin{array}{l}\text { Rossi LA, Torrati FG, Car- } \\
\text { valho EC, Manfrim A, Silva } \\
\text { DF [12]. }\end{array}$ & 2000 & $\begin{array}{l}\text { Diagnóstico de enfermagem do paciente no } \\
\text { período pós-operatório imediato. }\end{array}$ & $\begin{array}{l}\text { Lilacs; Rev Escola de Enfer- } \\
\text { magem - USP; 34(2):154- } \\
64 .\end{array}$ \\
\hline $\begin{array}{l}\text { Thomaz VA, Guidardello } \\
\text { EB [13]. }\end{array}$ & 2002 & $\begin{array}{l}\text { Sistematização da assistência de enfermagem: } \\
\text { problemas identificados pelos enfermeiros. }\end{array}$ & $\begin{array}{l}\text { BDENF; Nursing; 5(54):28- } \\
34 .\end{array}$ \\
\hline Andrade JS, Vieira MJ [14]. & 2005 & $\begin{array}{l}\text { Prática assistencial de enfermagem: problemas, } \\
\text { perspectivas e necessidade de sistematização. }\end{array}$ & $\begin{array}{l}\text { Lilacs; Rev Bras Enferma- } \\
\text { gem; 58(3):261-5. }\end{array}$ \\
\hline Backes DS, Schwatz E [15]. & 2005 & $\begin{array}{l}\text { Implementação da sistematização da assistência } \\
\text { de enfermagem: desafios e conquistas do ponto } \\
\text { de vista gerencial. }\end{array}$ & $\begin{array}{l}\text { Lilacs; Ciênc Cuid Saúde; } \\
4(2): 182-8 .\end{array}$ \\
\hline $\begin{array}{l}\text { Carvalho ARS, Matsuda } \\
\text { LM, Carvalho MS, Almeida } \\
\text { RMSSA, Schneider SLG } \\
\text { [16]. }\end{array}$ & 2006 & $\begin{array}{l}\text { Complicações no pós-operatório de revasculari- } \\
\text { zação miocárdica }\end{array}$ & $\begin{array}{l}\text { Lilacs; Ciênc Cuid Saúde; } \\
5(1): 50-59 .\end{array}$ \\
\hline $\begin{array}{l}\text { Rocha LA, Maia TF, Silva } \\
\text { LF [17]. }\end{array}$ & 2006 & $\begin{array}{l}\text { Diagnósticos de enfermagem em pacientes sub- } \\
\text { metidos à cirurgia cardíaca. }\end{array}$ & $\begin{array}{l}\text { Lilacs; Revista Brasileira de } \\
\text { Enfermagem; 59(3):321-6. }\end{array}$ \\
\hline $\begin{array}{l}\text { Lopes FL, Szewczyk MSC, } \\
\text { Lunardi VL, Santos SSC } \\
{[18] .}\end{array}$ & 2007 & $\begin{array}{l}\text { SAE como um novo fazer na atividade cuidativa } \\
\text { da enfermeira com base na complexidade de } \\
\text { Edgar Morin. }\end{array}$ & $\begin{array}{l}\text { BDENF; Cogitare Enferma- } \\
\text { gem; 12(1): 115-119. }\end{array}$ \\
\hline $\begin{array}{l}\text { Nascimento KC, Backes } \\
\text { DS, Koerich MS, Lorezini } \\
\text { [19]. }\end{array}$ & 2008 & $\begin{array}{l}\text { Sistematização da assistência de enfermagem: } \\
\text { vislumbrando um cuidado interativo, comple- } \\
\text { mentar e multiprofissional. }\end{array}$ & $\begin{array}{l}\text { Lilacs; Rev Esc Enfermagem } \\
\text { da USP; 42(4):643-8. }\end{array}$ \\
\hline $\begin{array}{l}\text { Truppel TC, Marineli JM, } \\
\text { Calixto RC, Peruzo AS, } \\
\text { Crozeta K [20]. }\end{array}$ & 2009 & $\begin{array}{l}\text { Sistematização da assistência de enfermagem } \\
\text { em Unidade de Terapia Intensiva }\end{array}$ & $\begin{array}{l}\text { Lilacs; Rev Esc Enfermagem } \\
\text { USP; 32(2):154-164. }\end{array}$ \\
\hline
\end{tabular}


Neste material foi realizada uma leitura exploratória, com o objetivo de fazer a caracterização inicial do problema, cuja classificação e definição constituem o primeiro estágio de toda pesquisa científica [10], sendo realizada também uma leitura seletiva que se qualifica pela determinaçáo do material que de fato interessa à pesquisa.

Em outro momento aconteceu uma leitura interpretativa deste material, que constitui a última etapa do processo de leitura de fontes bibliográficas através das quais podemos obter as respostas ao problema da pesquisa [21] e a análise temática dos artigos, que é a análise das informaçóes constantes de um documento [22].

\section{Resultados e discussão}

Através da análise e discussão dos dados coletados na bibliografia potencial, foi criado um quadro com os pontos de maior relevância encontrados nestes artigos que serviram de orientação para esta pesquisa.

Quadro IV - Descrição dos dados.

\begin{tabular}{|c|c|c|c|}
\hline Autor/ano & Título do artigo & Objetivo e metodologia & Principais considerações \\
\hline $\begin{array}{l}\text { Guerrieiro ALS, } \\
\text { Guimarães } \\
\text { HCQP, Maria } \\
\text { VLR } 2000 \text { [1 1]. }\end{array}$ & $\begin{array}{l}\text { Diagnósticos de } \\
\text { enfermagem do } \\
\text { paciente adulto no } \\
\text { primeiro pós- ope- } \\
\text { ratório de cirurgia } \\
\text { cardíaca. }\end{array}$ & $\begin{array}{l}\text { Objetivo: Identificar as categorias diag- } \\
\text { nósticas de enfermagem elaboradas } \\
\text { pelas enfermeiras para os pacientes } \\
\text { adultos, no primeiro pós-operatório de } \\
\text { cirurgia cardíaca. } \\
\text { Metodologia: Pesquisar de campo } \\
\text { realizado no Instituto Dante Pazzanese } \\
\text { de cardiologia hospital estadual, a } \\
\text { pesquisa foi realizada na URPO onde } \\
\text { foi feita a coleta e dados, tratamento } \\
\text { dos dados. }\end{array}$ & $\begin{array}{l}\text { Nesta pesquisa ficou constatado que } \\
\text { as enfermeiras ainda têm algumas } \\
\text { dificuldades na elaboração dos diag- } \\
\text { nósticos, tornando-os limitados em } 14 \\
\text { categorias, mas que existe uma preo- } \\
\text { cupação não apenas pelo fisiológico } \\
\text { na elaboração dos diagnósticos como } \\
\text { também com o psicológico e o social e } \\
\text { que existe a necessidade de um maior } \\
\text { aprofundamento em termos de conhe- } \\
\text { cimento científico. }\end{array}$ \\
\hline $\begin{array}{l}\text { Rossi L A, } \\
\text { Torrati FG, } \\
\text { Carvalho EC, } \\
\text { Manfrin A, } \\
\text { Silva DF } 2000 \\
{[12] .}\end{array}$ & $\begin{array}{l}\text { Diagnósticos de en- } \\
\text { fermagem em pa- } \\
\text { cientes no período } \\
\text { de pós-operatório } \\
\text { imediato. }\end{array}$ & $\begin{array}{l}\text { Objetivo: Identificar os diagnósticos de } \\
\text { enfermagem mais frequentemente de } \\
\text { pacientes operatórios imediato. } \\
\text { Metodologia: Estudo de campo cujos } \\
\text { dados coletados foram retirados de } \\
\text { uma ficha de avaliação pós-anestésica. }\end{array}$ & $\begin{array}{l}\text { O estudo fala sobre o diagnóstico } \\
\text { de enfermagem no pós-operatório } \\
\text { imediato de várias cirurgias e informa } \\
\text { os diagnósticos identificados pelas en- } \\
\text { fermeiras no período pós-anestésico e } \\
\text { que muitas vezes se usa do diagnóstico } \\
\text { médico para se formular os próprios } \\
\text { diagnósticos. No estudo foram identi- } \\
\text { ficados } 16 \text { diferentes diagnósticos que } \\
\text { quando passados para UTI podem } \\
\text { levar a elaboração de outros ou res- } \\
\text { postas para futuros desequilíbrios. }\end{array}$ \\
\hline $\begin{array}{l}\text { Thomas VA, } \\
\text { Guirdadello EB } \\
2002 \text { [13]. }\end{array}$ & $\begin{array}{l}\text { Sistematização da } \\
\text { assistência de en- } \\
\text { fermagem: proble- } \\
\text { mas identificados } \\
\text { pelos enfermeiros. }\end{array}$ & $\begin{array}{l}\text { Objetivo: Identificar os problemas } \\
\text { apresentados pelos enfermeiros de um } \\
\text { hospital escola do interior do Estado de } \\
\text { São Paulo quanto à operacionalização } \\
\text { da SAE. } \\
\text { Metodologia: Pesquisa de campo que } \\
\text { utilizou como instrumento de coleta de } \\
\text { dados um questionário semiestrutura- } \\
\text { do criado pelas autoras da pesquisa } \\
\text { com base na revisão de literatura e em } \\
\text { experiências e práticas. }\end{array}$ & $\begin{array}{l}\text { O estudo relata as dificuldades encon- } \\
\text { tradas pelos enfermeiros, dentre elas, } \\
\text { eles não são preparados, têm pouco } \\
\text { interesse, não têm conhecimento } \\
\text { necessário para colocar em prática o } \\
\text { método específico de sua especialida- } \\
\text { de e que não são responsáveis para } \\
\text { gerenciar as assistências, pois estão } \\
\text { habituados as rotinas e ao cumprimen- } \\
\text { to da prescrição médica. }\end{array}$ \\
\hline
\end{tabular}




\begin{tabular}{|c|c|c|c|}
\hline Autor/ano & Título do artigo & Objetivo e metodologia & Principais considerações \\
\hline $\begin{array}{l}\text { Andrade JS, } \\
\text { Vieira MJ / } \\
2005 \text { [14]. }\end{array}$ & $\begin{array}{l}\text { Prática assistencial } \\
\text { de enfermagem: } \\
\text { problemas, pers- } \\
\text { pectivas e necessi- } \\
\text { dade de sistemati- } \\
\text { zação. }\end{array}$ & $\begin{array}{l}\text { Objetivo: Relatar os principais desafios } \\
\text { e conquistas do ponto de vista geren- } \\
\text { cial no que tange à implementação da } \\
\text { Sistematização da Assistência de enfer- } \\
\text { magem (SAE), à luz de uma metodo- } \\
\text { logia problematizadora na realidade, } \\
\text { baseada em Paulo Freire. } \\
\text { Metodologia: trata-se de um estudo } \\
\text { qualitativo descritivo para subsidiar a } \\
\text { implantação da SAE em um hospital } \\
\text { universitário. }\end{array}$ & $\begin{array}{l}\text { Os resultados desta pesquisa identifi- } \\
\text { caram as atividades, percepção sobre } \\
\text { a enfermagem e cliente, conhecimento } \\
\text { e aplicação do processo de enfer- } \\
\text { magem e decorrentes da assistência } \\
\text { não sistematizada. Os resultados } \\
\text { identificaram que as atividades dos } \\
\text { enfermeiros baseiam-se no tecnicismo } \\
\text { e na administração do serviço que a } \\
\text { enfermagem foi relacionada com o } \\
\text { atendimento às necessidades humanas } \\
\text { básicas e holismo, sendo o cliente de- } \\
\text { finido como o indivíduo que necessita } \\
\text { de cuidados; que a maioria conhecia } \\
\text { na teoria o processo de Enfermagem, } \\
\text { mas não o aplicou na prática. }\end{array}$ \\
\hline $\begin{array}{l}\text { Backes DS, } \\
\text { Schwart E } \\
2005 \text { [5]. }\end{array}$ & $\begin{array}{l}\text { Implementação } \\
\text { da sistematização } \\
\text { da assistência de } \\
\text { enfermagem: de- } \\
\text { safios e conquistas } \\
\text { do ponto de vista } \\
\text { gerencial. }\end{array}$ & $\begin{array}{l}\text { Objetivos: Relatar os principais desafios } \\
\text { e conquistas do ponto de vista geren- } \\
\text { cial no que tange à implementação } \\
\text { da sistematização da assistência de } \\
\text { enfermagem (SAE). } \\
\text { Metodologia: problematizadora da } \\
\text { realidade, baseada em Paulo Freire. }\end{array}$ & $\begin{array}{l}\text { A experiência demonstra que mesmo } \\
\text { com a deficiência de recursos huma- } \\
\text { nos, a descrença e o despreparo de } \\
\text { muitos profissionais, o processo de } \\
\text { implantação do SAE alcançou êxito à } \\
\text { medida que se desenvolveram estraté- } \\
\text { gias gerenciais dialógicas e reflexivas } \\
\text { no coletivo, capazes de problematizar } \\
\text { as ações reducionistas de um fazer me- } \\
\text { ramente técnico e burocratizado. }\end{array}$ \\
\hline $\begin{array}{l}\text { Carvalho ARS, } \\
\text { Matsuda LM, } \\
\text { Carvalho MSS, } \\
\text { Almeida RMS- } \\
\text { SA, Schneider } \\
\text { DSLG } 2006 \\
\text { [16]. }\end{array}$ & $\begin{array}{l}\text { Complicações no } \\
\text { pós-operatório de } \\
\text { revascularização } \\
\text { miocárdica. }\end{array}$ & $\begin{array}{l}\text { Objetivo: Investigar os principais tipos } \\
\text { de complicações ocorridas no perí- } \\
\text { odo pós-operatório de RVM em } 119 \\
\text { indivíduos. } \\
\text { Metodologia: estudo quantitativo, des- } \\
\text { critivo, transversal realizado por meio } \\
\text { de consulta ao banco de dados do } \\
\text { serviço de cirurgia cardiovascular de } \\
\text { um hospital geral de Cascavel, PR. }\end{array}$ & $\begin{array}{l}\text { O estudo fala sobre a incidência de } \\
\text { complicações em RVM, que são muito } \\
\text { maiores devido a diversos fatores, e as } \\
\text { doenças podendo gerar uma possível } \\
\text { RVM. }\end{array}$ \\
\hline $\begin{array}{l}\text { Rocha LA, } \\
\text { Maia TF, Silva } \\
\text { LF } 2006 \text { [17]. }\end{array}$ & $\begin{array}{l}\text { Diagnósticos de } \\
\text { enfermagem em } \\
\text { pacientes subme- } \\
\text { tidos à cirurgia } \\
\text { cardíaca. }\end{array}$ & $\begin{array}{l}\text { Objetivos: identificar diagnósticos de } \\
\text { enfermagem segundo a taxonomia II } \\
\text { da NANDA e intervenções de enferma- } \\
\text { gem segundo a NIC associados aos } \\
\text { resultados de enfermagem segundo a } \\
\text { NOC. } \\
\text { Metodologia: pesquisa descritiva explo- } \\
\text { ratória do tipo transversal. }\end{array}$ & $\begin{array}{l}\text { Neste estudo foram identificados } 15 \\
\text { diagnósticos de enfermagem segundo } \\
\text { a taxonomia II NANDA sendo } 15 \text { de } \\
\text { maior prevalência, nos mostra também } \\
\text { que a partir destes diagnósticos, foram } \\
\text { identificadas as principais intervenções } \\
\text { e resultados de acordo com a ligação } \\
\text { entre NANDA, NIC, NOC. }\end{array}$ \\
\hline
\end{tabular}




\begin{tabular}{|c|c|c|c|}
\hline Autor/ano & Título do artigo & Objetivo e metodologia & Principais considerações \\
\hline $\begin{array}{l}\text { Lopes F L, } \\
\text { Szewczyk MSC, } \\
\text { Lunardi VL, } \\
\text { Santos SSC } \\
2007 \text { [18]. }\end{array}$ & $\begin{array}{l}\text { SAE como um novo } \\
\text { fazer na atividade } \\
\text { cuidativa da enfer- } \\
\text { meira com base na } \\
\text { complexidade de } \\
\text { Edgar Morin. }\end{array}$ & $\begin{array}{l}\text { Objetivo: Realizar uma aproximação } \\
\text { da SAE com a complexidade de Edgar } \\
\text { Morin. } \\
\text { Metodologia: reflexão teórica. }\end{array}$ & $\begin{array}{l}\text { É uma reflexão que fala da importân- } \\
\text { cia do cuidar para ser humano e as } \\
\text { influências que a SAE traz enquanto } \\
\text { ferramenta indispensável ao cuidado } \\
\text { de enfermagem, ressaltando que cada } \\
\text { indivíduo sob o cuidado de enferma- } \\
\text { gem apresenta especificidade e mul- } \\
\text { tidimensionalidade, além de destacar } \\
\text { o ser humano como um ser complexo } \\
\text { tornando possível sua aproximação } \\
\text { com os princípios da complexidade } \\
\text { proposta por Edgar Morin. }\end{array}$ \\
\hline $\begin{array}{l}\text { Nascimento } \\
\text { KC, Backes } \\
\text { DS, Koerich } \\
\text { MS, Erdmann } \\
\text { AL } 2008 \text { [19]. }\end{array}$ & $\begin{array}{l}\text { Sistematização } \\
\text { da assistência } \\
\text { de enfermagem: } \\
\text { vislumbrando um } \\
\text { cuidado interativo, } \\
\text { complementar e } \\
\text { multiprofissional }\end{array}$ & $\begin{array}{l}\text { Objetivo: Compreender o significado } \\
\text { da sistematização da assistência de } \\
\text { enfermagem para os profissionais de } \\
\text { saúde. } \\
\text { Metodologia: Trata-se de um projeto } \\
\text { amplia do, qualitativo, que se utilizou } \\
\text { como referencial metodológico a Teoria } \\
\text { fundamentada nos dados, que foram } \\
\text { coletados por meio de entrevistas com } \\
\text { três grupos amostrais, totalizando } 15 \\
\text { profissionais de saúde. }\end{array}$ & $\begin{array}{l}\text { Nesse artigo é discutido o fenôme- } \\
\text { no verificando a necessidade de um } \\
\text { processo interativo, complementar e } \\
\text { multiprofissional. A SAE é parte de um } \\
\text { processo que vem sendo desenvolvido } \\
\text { ao longo do tempo por enfermeiros } \\
\text { comprometidos em melhorar cada vez } \\
\text { mais o cuidado prestado ao pacien- } \\
\text { te, pois vislumbramos a necessidade } \\
\text { do cuidar interativo, complementar e } \\
\text { multiprofissional. }\end{array}$ \\
\hline $\begin{array}{l}\text { Truppel TC, } \\
\text { Meier MJ, Ca- } \\
\text { lixto RC, Peru- } \\
\text { zo AS, Crozeta } \\
\text { K } 2009 \text { [20]. }\end{array}$ & $\begin{array}{l}\text { Sistematização da } \\
\text { assistência de en- } \\
\text { fermagem em UTI }\end{array}$ & $\begin{array}{l}\text { Objetivo: Identificar as situações de } \\
\text { saúde - doença e as necessidades de } \\
\text { cuidados de enfermagem, bem como } \\
\text { subsidiar as intervenções de promoção, } \\
\text { prevenção, recuperação e reabilita- } \\
\text { ção da saúde do indivíduo, família e } \\
\text { comunidade. Reestruturar o SAE em } \\
\text { uma UTI; elencar os diagnósticos e as } \\
\text { prescrições de enfermagem; validar as } \\
\text { etapas da SAE e subsidiar a estrutura- } \\
\text { ção de um protocolo para operaciona- } \\
\text { lização do SAE. } \\
\text { Metodologia: Pesquisa de campo com } \\
\text { a participação de 06 enfermeiros da } \\
\text { UTI a qual se dividiu em descrição da } \\
\text { prática de enfermagem e transcrição } \\
\text { dos diagnósticos de enfermagem. } \\
\text { Construção de um protocolo de diag- } \\
\text { nósticos de enfermagem; Determina- } \\
\text { ção das prescrições de enfermagem; } \\
\text { construção de normas, rotinas e proce- } \\
\text { dimentos e aspectos éticos. }\end{array}$ & $\begin{array}{l}\text { Foram identificados } 29 \text { diagnósticos } \\
\text { NANDA e } 38 \text { prescrições, relata a im- } \\
\text { portância dos registros de enfermagem } \\
\text { que sejam de qualidade e internacio- } \\
\text { nalmente reconhecidos, e que possam } \\
\text { ser interpretados pelas equipes multi- } \\
\text { disciplinares. }\end{array}$ \\
\hline
\end{tabular}


A partir desta análise foram coletados os dados para a criação de duas categorias que serão apresentadas a seguir.

\section{Benefícios encontrados com o uso da SAE para os pacientes em pós-operatório de cirurgia cardíaca}

Com a análise dos dados dos artigos selecionados, podemos destacar as vantagens da institucionalização da SAE para os pacientes em pós-operatório de cirurgia cardíaca como sendo benéfica para os cuidados prestados.

Segundo a análise de Andrade [14], o enfermeiro, ao planejar a assistência, garante sua responsabilidade junto ao cliente assistido, uma vez que o planejamento permite diagnosticar suas necessidades. Dessa forma, ficam garantidas a prescrição adequada dos cuidados, a supervisão do desempenho do pessoal, a avaliação dos resultados e a qualidade da assistência.

Andrade [14] acredita também que a aplicação do processo de enfermagem é baseada em princípios e regras que são conhecidas por promover cuidado de enfermagem eficiente, proporcionar ao enfermeiro a possibilidade da prestação de cuidados individualizados, centrados nas necessidades humanas básicas, além de nortear tomadas de decisão em diversas situaçôes vivenciadas pelo profissional enquanto gerenciador da equipe de enfermagem.

Backes [15], afirma que a SAE representa a conquista de um "novo espaço" isto é, um novo modo de ser e agir. Segundo ele, a enfermagem necessita ser questionada à luz de metodologias problematizadoras, a fim de não se constituir em mais uma alternativa frustrada, ou um processo meramente normativo e/ou legal. Como resultado, o profissional poderá chegar a ser sujeito agente de mudança por meio da ação-reflexão acerca de sua realidade concreta.

De acordo com Rocha [16], conhecer os problemas de saúde de um grupo de pessoas com características comuns, poderá também direcionar a assistência de enfermagem ao fornecer subsídios para a elaboraçáo de planos de cuidados, implementação das intervençóes e avaliação de acordo com as necessidades do paciente. Este trabalho pode vir a ser o início de um estudo da validação por meio da prática clínica, ou ainda, constituir subsídio para o enfermeiro no cuidado de pacientes no pós-operatório de cirurgia cardíaca.
Para Carvalho [17], o conhecimento dos tipos de problemas no pós-operatório de revascularização miocárdica é de suma importância, principalmente para a enfermagem, visto que favorece a implementação de açóes pró-ativas ao paciente, no sentido de prevenir danos e/ou sequelas. $\mathrm{O}$ enfermeiro deve participar do cuidado e orientaçáo do indivíduo desde o momento em que ele toma ciência da necessidade do procedimento, pois neste contexto o enfermeiro, bem instruído e conhecedor da realidade em que atua, tem mais chances de contribuir para a boa evolução do paciente em pós-operatório, no sentido de promover uma recuperação melhor e mais rápida.

Segundo Lopes [18], SAE é entendida como uma possibilidade de um melhor cuidado de enfermagem e sua implantação pode contribuir para definir a enfermagem como ciência, elevando nossa credibilidade profissional perante nossos pares, outros profissionais e a população. Quando a aplicamos, temos melhores condiçóes para identificar, compreender e descrever como o cliente está reagindo frente a seus processos vitais e seus problemas, e quais cuidados profissionais devem ser implementados.

Sob o ponto de vista de Nascimento [19], a implementação de um modelo e/ou uma fórmula predeterminada de assistência náo é garantia de maior qualidade na assistência em saúde. É preciso também que se estabeleçam novas e mais complexas relaçóes e interaçóes profissionais para apreender o ser humano de forma ampla e integral.

Segundo Nascimento [18], com base nessas e outras ideias, tornou-se cada vez mais incisivo o desejo de compreender a SAE a partir de novos referenciais, capazes de ampliar o campo de visão para além das fórmulas prescritas e normativas e, sobretudo, para além dos modelos formalmente instituídos como norteadores de uma assistência centrada no ser humano. A sistematização da assistência de enfermagem, enquanto processo organizacional é capaz de oferecer subsídios para o desenvolvimento de métodos/metodologias interdisciplinares e humanizadas de cuidado.

Conforme afirma Truppel [22], diante do cuidado altamente especializado e complexo que o enfermeiro desenvolve em uma unidade de terapia intensiva, a sistematização e a organizaçáo do seu trabalho e, por conseguinte, do trabalho da equipe de enfermagem, mostram-se imprescindíveis para uma assistência de qualidade com eficiência e eficácia. 
Conforme foi visto na análise dos artigos, a SAE é de extrema importância para o paciente que precisa de cuidados individualizados de enfermagem, evitando, assim, problemas nos pós-operatórios de cirurgia cardíaca. A SAE também possibilita ao enfermeiro ter um olhar mais amplo daquele cliente, podendo assim intervir mais eficazmente, atendendo às suas necessidades, com cuidados cada vez mais individualizados em todos os aspectos.

\section{Dificuldades encontradas pelos enfermeiros ao usar a SAE como instrumento do cuidado}

De acordo com os achados na análise dos artigos, podemos observar que muitos enfermeiros encontram dificuldades na utilizaçáo da SAE, o que pode tornar o cuidado ineficiente, criando, com isso, um fator desencadeante de futuros problemas e complicaçôes que podem surgir no pós-operatório destes clientes submetidos à cirurgia cardíaca, causando a demora em sua recuperaçáo e, às vezes, o agravamento do quadro.

Conforme Guerrieiro [11], a implementação dos diagnósticos de enfermagem na unidade de recuperação pós-operatória tem sido uma experiência difícil, pois tem exigido mudanças na postura dos profissionais que se mostravam mais envolvidos com a área tecnológica.

Segundo Thomaz [13], dentre essas dificuldades, destacam-se a falta de conhecimento em realizar as diferentes etapas do processo de enfermagem, o desconhecimento de como realizar o exame físico, a prática de enfermagem vinculada ao cumprimento das atividades burocráticas e técnicas, (o detrimento do processo de enfermagem), falta de preparo e a não-visualizaçáo do enfermeiro como responsável para gerenciar a assistência de enfermagem, pois está habituado a rotinas e ao cumprimento da prescrição médica.

Thomaz [13] acredita que outras dificuldades apontadas foram as falhas na implantação da assistência sistematizada e no acompanhamento periódico e correto das atividades (que podem resultar em perda de estímulo), razão da existência de dúvidas e do seu náo-esclarecimento, falta de pessoal que pode gerar desmotivação, desconhecimento da lei de exercício profissional pelos enfermeiros, falta de liderança desse profissional que, por sua vez, acaba não assumindo o papel de educador, e o número excessivo de pacientes para prescrever e evoluir num único plantão.
$\mathrm{Na}$ análise de Andrade [14], os enfermeiros, devido ao modelo biomédico hegemônico, tanto nas escolas de formaçáo profissional quanto nas instituiçôes hospitalares, tendem a valorizar o tecnicismo durante a assistência, desconsiderando, na maioria das vezes, os aspectos individuais e emocionais do cliente. Essa dificuldade de compreensão sobre as atividades administrativas do enfermeiro, visto que a função administrativa é essencial para que a assistência seja prestada, não havendo como desarticulá-las, é outro ponto a ser considerado.

Sob o relato de Andrade [14], para o enfermeiro administrar é preciso saber prestar o cuidado, ou seja, ele não pode administrar sem assistir. $\mathrm{O}$ fato de não conhecerem as necessidades do cliente de forma holística, devido a não utilizaçáo do processo de enfermagem SAE, interfere negativamente na interação enfermeiro/cliente, reduzindo as possibilidades de uma assistência comprometida com a qualidade.

Outro aspecto evidenciado por Andrade [14] foi a desorganização do serviço gerada pelas diferentes formas de conduta profissional na assistência, falta de padronizaçáo dos procedimentos, inexistência de normas e rotinas, bem como a náo utilização de uma metodologia de assistência de enfermagem.

Do ponto de vista de Andrade [14], ainda como problema decorrente, encontra-se o conflito dos papéis. Os enfermeiros dividem-se entre o desejo de prestar assistência ao cliente e as cobranças administrativas da instituição. Em relação ao desgaste de recursos humanos encontra-se outro problema, pois no momento em que percebe o mau funcionamento e baixo rendimento pela sua equipe de trabalho, passa a desacreditar no seu potencial enquanto líder e gerente, produzindo sensaçóes de impotência e frustração que, por sua vez, proporciona maior desgaste físico e, consequentemente, má produtividade, quantitativa e qualitativamente, na prestação de cuidados.

Finalizando a análise de Andrade [14], existe a perda de tempo, já que em um ambiente de trabalho onde não existe planejamento das atividades nem determinaçáo de prioridades há uma perda de tempo significativa no processo de gerenciamento, levando os profissionais a refazerem as atividades realizadas sem êxito. Assim perde-se tempo em resolver problemas inerentes a outros profissionais da equipe e, quando realmente se trata de atividades que competem ao enfermeiro, este náo mais dispóe de tempo para realizá-las. 
Segundo Rocha [17], é importante atentar para a queixa da dor do paciente, pois a falta do cuidado necessário poderá desencadear ansiedade, com alteraçóes de sinais vitais e desperdício de energia.

Após essa análise detalhada das problemáticas encontradas pelo enfermeiro, pode-se perceber que a enfermagem precisa ainda de preparo para que possa desenvolver de modo eficiente a sistematização da assistência de enfermagem, pois mesmo reconhecendo seus benefícios evidentes, o que falta ao enfermeiro é o preparo, uma vez que estão ainda muito focados no desenvolvimento técnico e no cumprimento da prescrição médica.

O fato de estarem sobrecarregados também faz com que náo consigam desenvolver um plano de cuidados voltados para as necessidades reais dos pacientes em pós-operatório de cirurgia cardíaca, no sentindo de contribuir para uma boa evolução e recuperação, com o intuito de obter a alta hospitalar o mais precocemente possível.

\section{Conclusão}

A sistematização da assistência de enfermagem, enquanto processo organizacional é capaz de oferecer subsídios para o desenvolvimento de métodos/ metodologias interdisciplinares e humanizadas de cuidado.

Conhecer os problemas de saúde de um grupo de pessoas, bem como características comuns, permite o planejamento da assistência a fim de diagnosticar as necessidades do cliente, garantindo a prescrição adequada dos cuidados individualizados, centrada nas necessidades humanas básicas.

Dessa forma, o enfermeiro bem preparado e conhecedor da realidade em que atua, tem mais chances de contribuir para uma evolução satisfatória do paciente em pós-operatório, no sentido de recuperar com qualidade e obter a alta hospitalar o mais precocemente possível.

Através da realização desta pesquisa, buscamos atingir nosso objetivo de descrever os benefícios da sistematizaçáo da assistência de enfermagem no pós-operatório de cirurgia cardíaca para os clientes (assunto bastante atual), porém pouco explorado em literatura.

Analisamos também alguns empecilhos impostos à $\mathrm{SAE}$, como a dificuldade de alguns enfermeiros em se adaptar a esse método e a sobrecarga de trabalho.
A falta de conhecimentos em realizar as diferentes etapas do processo de enfermagem, a falta de preparo, a não-visualização da enfermagem como responsável por gerenciar a assistência e o fato de não conhecer as necessidades do cliente de forma holística, interferem negativamente na interação enfermeiro/paciente, comprometendo a qualidade da assistência.

O profissional quando não percebe, imediatamente, o mau funcionamento e o baixo rendimento de sua equipe, sofre uma sobrecarga de trabalho e passa a desacreditar no seu potencial enquanto líder e gerente. A sensação de impotência e frustração causada por tal conjuntura proporciona um desgaste físico e, consequentemente, má produtividade, quantitativa e qualitativamente, na prestação de cuidados.

Percebe-se, portanto, a necessidade de atuação eficaz das instituiçóes de saúde para que ofereçam condiçóes satisfatórias para o desenvolvimento deste trabalho, favorecendo a diminuição do tempo de hospitalização deste paciente, diminuindo, consequentemente, os custos.

Diante dessa realidade, podemos concluir que a sistematização da assistência de enfermagem no pós-operatório de cirurgia cardíaca é muito importante para que o cliente tenha uma recuperação rápida e plena, e que a equipe, com a implantação da SAE, consiga tornar seu trabalho mais organizado e eficiente.

Para tal, é necessário que o enfermeiro mude sua postura e se envolva mais, desempenhando suas funçóes, centralizadas nos cuidados individualizados de acordo com cada necessidade do paciente.

Deve-se considerar, entretanto, que, para a aplicação da sistematização da assistência de enfermagem é necessário que haja a melhoria da qualificação destes profissionais. Há que se dar um enfoque maior ao assunto nos cursos de nível superior, pois constatamos que muitos profissionais saem das universidades sem o preparo necessário para desenvolver este tipo de trabalho com o cliente.

\section{Referências}

1. Cianciarullo TI, ed. Instrumentos básicos para cuidá-lo: um desafio para a qualidade de assistência. São Paulo: Atheneu; 2005.

2. Horta WA. Processo de Enfermagem. Sáo Paulo: EPU; 1979.

3. Conselho Federal de Enfermagem (BR). Resolução no. 272/2002. Dispóe sobre a sistematização da assistência 
de enfermagem - SAE - nas Instituiçóes de Saúde Brasileiras. [citado 2009 Set 27]. Disponível em URL: http://www.portalcofen.com.br

4. Chutannure M. Sistematizaçáo da Assistência de Enfermagem: Guia prático. Rio de Janeiro: Guanabara Koogan; 2007.

5. Smetzer SC, Bare BG. Tratado de Enfermagem Médico Cirúrgico. 10a ed. Rio de Janeiro: Guanabara Koogan; 2005.

6. NANDA. Diagnósticos de Enfermagem da NANDA: definiçóes e classificaçóes 2007 e 2008. Porto Alegre: Artmed; 2008.

7. Knobel E, Aguiar LF, Ghiotto JL. Condutas no paciente grave. Rio de Janeiro: Atheneu; 2006. p.1573-84.

8. Galantier J, Auler JR. Terapia intensiva adulto e pediátrico. São Paulo: Revinter; 2003. p. 693-703.

9. Mertens RSK, Fumanga M. Como elaborar projetos de pesquisa: linguagem e método. Rio de Janeiro: FGV; 2007.

10. Koche JC. Fundamentos de metodologia científica, teoria da ciência e prática da pesquisa. Petrópolis: Vozes; 1997.

11. Guerriero ALS, Guimarães HCQCP, Maria VLR. Diagnóstico de enfermagem do paciente adulto no primeiro pós-operatório de cirurgia cardíaca. Acta Paul Enfermagem 2000;13(2):59-67.

12. Rossi LA, Torrati FG, Carvalho EC, Manfrim A, Silva DF. Diagnóstico de enfermagem do paciente no período pós-operatório imediato. Rev Esc Enfermagem USP 2000;34(2):154-64.

13. Thomaz VA, Guidardello EB. Sistematização da assistência de enfermagem: problemas identificados pelos enfermeiros. Nursing 2002;5(54):28-34.
14. Andrade JS, Vieira MJ. Prática Assistencial de Enfermagem: problemas, perspectivas e necessidade de sistematização. Rev Bras Enfermagem 2005;58(3):261-5.

15. Backes DS, Schwatz E. Implementação da Sistematização da Assistência de Enfermagem: desafios e conquistas do ponto de vista gerencial. Ciênc Cuid Saúde 2005;4(2):182-8.

16. Carvalho ARS, Matsuda IM, Carvalho MS, Almeida RMSSA, Schneider DSLG. Complicaçôes no pós-operatório de revascularizaçáo miocardica. Ciênc Cuid Saúde 2006;5(1):50-9.

17. Rocha L, Maia TF, Silva LF. Diagnósticos de enfermagem em pacientes submetidos à cirurgia cardíaca. Rev Bras Enfermagem 2006;59(3):321-6.

18. Lopes FL, Szewczyk MSC, Lunardi VL, Santos SSC. SAE como um novo fazer na atividade cuidativa da enfermeira com base na complexidade de Edgar Morin. Cogitare Enfermagem 2007;12(1):115-9.

19. Nascimento KC, Backes DS, Koerich MS, Erdmann AL. Sistematização da Assistência de Enfermagem: vislumbrando um cuidado interativo, complementar e multiprofissional. Rev Esc Enfermagem USP 2008;42(4):643-8.

20. Truppel TC, Marineli JM, Calixto RC, Peruzo AS, Crozeta K. Sistematização da assistência de enfermagem em unidade de terapia intensiva. Rev Escola Enfermagem USP 2009;32(2):154-64.

21. Gil AC. Como elaborar projetos de pesquisa. $4 \mathrm{a}$ ed. São Paulo: Atlas; 2007.

22. Severino AJ. Metodologia do trabalho científico. São Paulo: Cortez; 2007. 